\title{
Correction to: The Development of Evaluation Algorithm for Blood Infection Degree
}

\author{
Chang-Hyun Kim ${ }^{1} \cdot$ Lee-Ku Kwac ${ }^{2} \cdot$ Hong-Gun $\mathrm{Kim}^{3}$
}

Published online: 5 October 2017

(C) The Author(s) 2017

\section{Correction to: Wireless Pers Commun DOI 10.1007/s11277-017-4136-0}

The Acknowledgements section in the original publication is incorrect. The correct Acknowledgements section is given here.

Acknowledgements This work was supported by the National Research Foundation of Korea (NRF) grant funded by the Korea government (MSIP) (No. 2017R1A2B4009646). It was also supported by Basic Science Research Program through the National Research Foundation of Korea (NRF) funded by the Ministry of Education (No. 2016R1A6A03012069).

The online version of the original article can be found under doi:10.1007/s11277-017-4136-0.

Hong-Gun Kim

hkim@jj.ac.kr

Chang-Hyun Kim

tobesure@jj.ac.kr

Lee-Ku Kwac

kwac29@jj.ac.kr

1 Institute of Carbon Technology, Jeonju University, 303 Cheonjam-ro, Wansan-gu, Jeonju, Jeollabuk-do 55069, Korea

2 Department of Manufacturing and Design Engineering, Jeonju University, 303 Cheonjam-ro, Wansan-gu, Jeonju, Jeollabuk-do 55069, Korea

3 Department of Mechanical and Automotive Engineering, Jeonju University, 303 Cheonjam-ro, Wansan-gu, Jeonju, Jeollabuk-do 55069, Korea 